\title{
Locality, QED and Classical Electrodynamics
}

\author{
Dirk Kreimer* \\ Mainz University
}

October 22, 2018

\begin{abstract}
We report on some conceptual changes in our present understanding of Quantum Field Theory and muse about possible consequences for the understanding of $v>c$ signals.
\end{abstract}

\section{Introduction}

This paper is a musing about the role which locality might have to play in the understanding of the phenomena which we gathered to discuss at this conference. Locality has always been the crucial property in one's approach to Quantum Field Theory (QFT). Mainly, there are two lines of thought. One is to maintain locality. One then can follow the standard derivation of Feynman rules and obtains a QFT which suffers from UV-divergences. These divergences unavoidably reflect the presence of local quantum fields, operator-valued distributions which, when we clumsily try to multiply them at coinciding space-time points, produce these UV-divergences.

A QFT as popular as Quantum Electrodynamics is the archetypical example of such a theory. Its merits are indisputable though. No experimental fact which is in conflict with its predictions (and with its natural successor at higher energies, the Standard Model of particle physics) has yet been found, and the theory is rightly praised for the accuracy with which it describes nature.

Nevertheless, the price is high. The theory is formulated in form of a highly dubious perturbative expansion: not only do we have to ignore that this expansion in the coupling constant is by no means convergent (even its Borel transform is in doubt), but we also are confronted with the fact that at each order in this expansion we have to manage ill-defined mathematical expressions, due to the very presence of UV-divergences, and thus being a direct consequence of working with a local QFT. Physicists found a way how to handle these ill-defined

*dirk.kreimer@uni-mainz.de 
expressions, known as renormalization. Renormalization is often regarded at best a technical rather than an illuminating device.

But their guilty conscience, for working with a priori ill-defined quantities, often let physicists to abandon local QFT, by either turning away completely from these issues, or, and this is the other line of thought, trying to seek recourse in other solutions. Most prominent here is string theory and its many generalizations, which all replace the point-particle concept by somewhat extended objects, and henceforth avoid UV divergences. Unfortunately, these approaches so far fail dramatically to describe nature.

Thus, it is no surprise that a theory like QED was rarely taken as a guide in subtle conceptual questions, its own foundations seemingly being built on unsecure ground.

This seems to change recently. What we see emerging from the art of calculation of Feynman diagrams is the mathematics which enables us to reconcile the practical successes of local QFT with a mathematical foundation which is well-defined and promising.

In the next section, I will describe some of the features of this mathematical backbone of a local QFT and in the final section draw some conclusions concerning the topic of this workshop.

But before I stretch your patience with a rush through the math of local QFT, let me first give you a hint how a QFT might have something to say of relevance for what Prof. Low called the Nimtz anomaly [1] in his talk.

What Low showed us was how one can establish a simple calculus which correctly describes the data delivered by the Nimtz experiment [2]. His calculus showed that the Nimtz anomaly is completely determined by height and length of the barrier, and showed the Nimtz anomaly as a function of these two variables.

To have a manageable calculus, Low did a step which is everyday practice for someone working in local Quantum Field Theory: he eliminated negligible contributions which stem from unmeasurable internal high frequencies, a step which was mandatory to arrive at the desired calculus, and which was justified by the fact that the abandoned contributions were small with repect to the problem in question. Nevertheless it is far reaching, as such tiny modifications can have drastical conceptual consequences.

To me, it seems that it is indeed a well-posed question to ask what the precise message is which local QFT has in store for us in the context of conceptualization of notions like signal, frequency band limitations and so on. Admittedly, one can regard classical field theory as detached from local QFT for such classical phenomena as the propagation of a microwavesignal to a (quenched) waveguide.

But then, classical electrodynamics ought to be the limit of QED in some yet to be defined sense, and thus, if QED lives in a universe in which a frequency band limitation, more usually dubbed UV cut-off in the jargon of a practitioner of QFT, is unavoidable, it might be that there is a message which QFT has indeed in store for us: to be careful to find those solutions to the classical equa- 
tions of motions which remain stable under negligible modifications of internal (unobserved) high frequency components.

So what is then the conceptual backbone which underlies local QFT and its need for a cut-off at high frequencies, which became covariant renormalization in modern terminology?

\section{The concept of renormalization}

The basic idea is to trace back the concept of renormalization to the structure of a Hopf algebra. In so doing, one recovers that the process of renormalization has a well-defined mathematical meaning and is related to the study of the diffeomorphism group of spacetime [3].

Fig.(11) summarizes some basic notions. Especially, we indicate how to calculate the coproduct of a rooted tree. In so doing, we cut the tree in pieces. Each of these pieces will correspond to a local counterterm in an analytic expression which renders a certain subdivergence finite.

In particular, this Hopf algebra has an antipode, which maps to Feynman diagrams as their local counterterm.

Fig.(2) gives a diagrammatic explanation of this Hopf algebra, and shows how the combinatorics of the renormalization procedure derives from this Hopf algebra.

The universal structure of the Hopf algebra [3] guarantees that it can be applied as long as we can render a theory finite by local counterterms: locality of the theory and its Hopf algebra structure are two sides of the same coin.

Let us also stress that the appearance of this Hopf algebra structure in QFT establishes a link to recent developments in mathematics. Quite the same Hopf algebra turned up in the work of Alain Connes and Henri Moscovici on the noncommutative index theorem. The relation is by now made precise, and puts local QFT on a firm mathematical ground [3].

The renormalized Green function can be recovered by the map

$$
\Gamma \rightarrow m\left[\left(S_{R} \otimes i d\right)(\phi \otimes \phi) \Delta\left(T_{\Gamma}\right)\right] .
$$

This map associates to the Feynman graph $\Gamma$ the renormalized Feynman integral [3].

Indeed, this map can be written as

$$
\Gamma \rightarrow \Gamma-\tau_{R}(\Gamma)+\left(i d-\tau_{R}\right)\left[\sum_{\gamma \subset \Gamma} Z_{\gamma} \Gamma / \gamma\right],
$$

where

$$
S_{R}\left(\phi\left(T_{\gamma}\right)\right) \equiv Z_{\gamma}=-\tau_{R}(\gamma)-\tau_{R}\left[\sum_{\gamma^{\prime} \subset \gamma} Z_{\gamma^{\prime}} \gamma / \gamma^{\prime}\right] \text {, }
$$



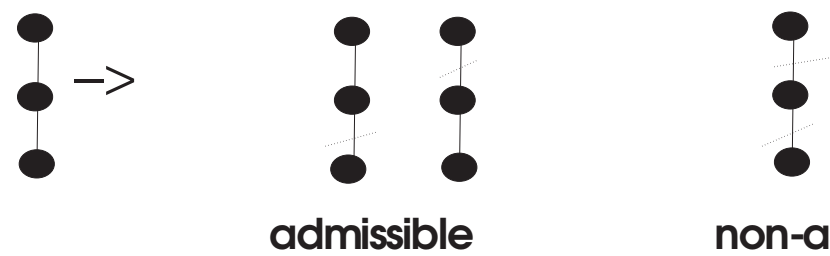

non-adm.

Cuts
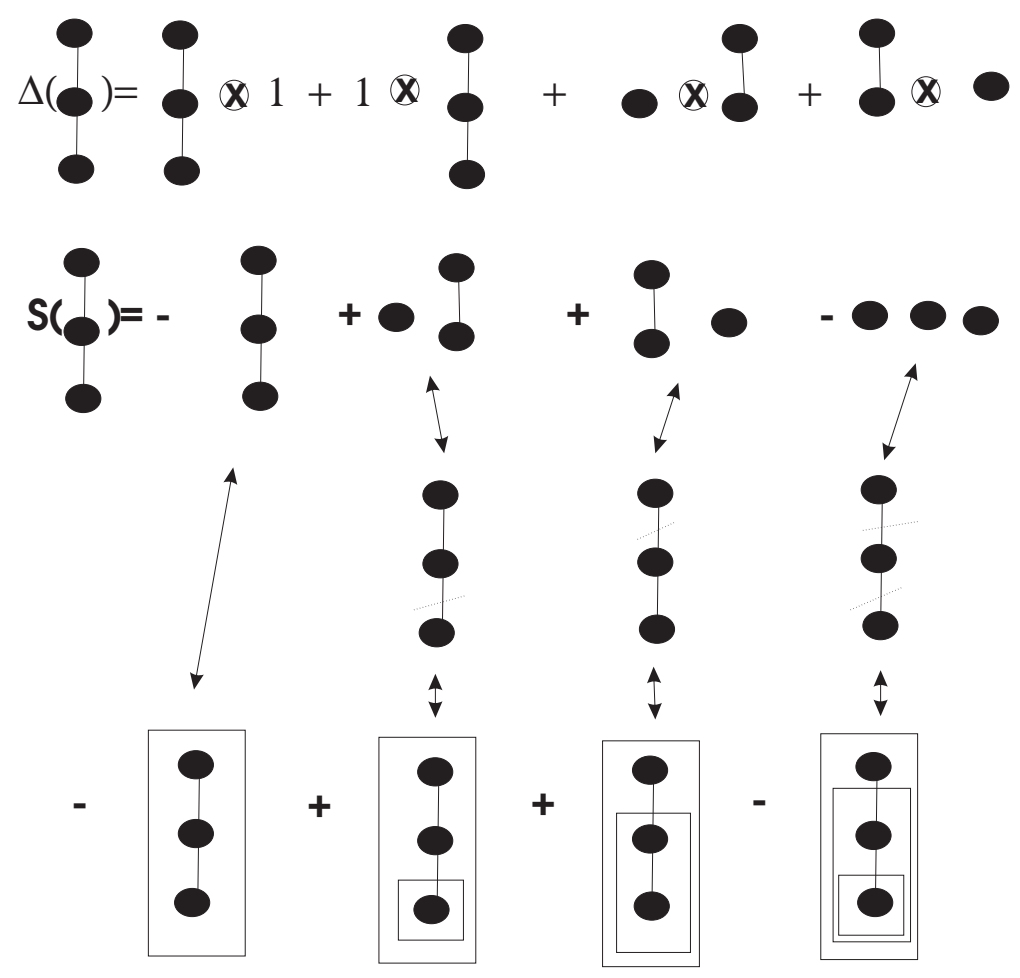

Figure 1: The Hopf algebra of rooted trees. We define it using admissible cuts on the trees, and give the coproduct $\Delta$ in terms of admissible cuts. An admissible cut allows for at most one single cut in any path from any vertex to the root [3]. 

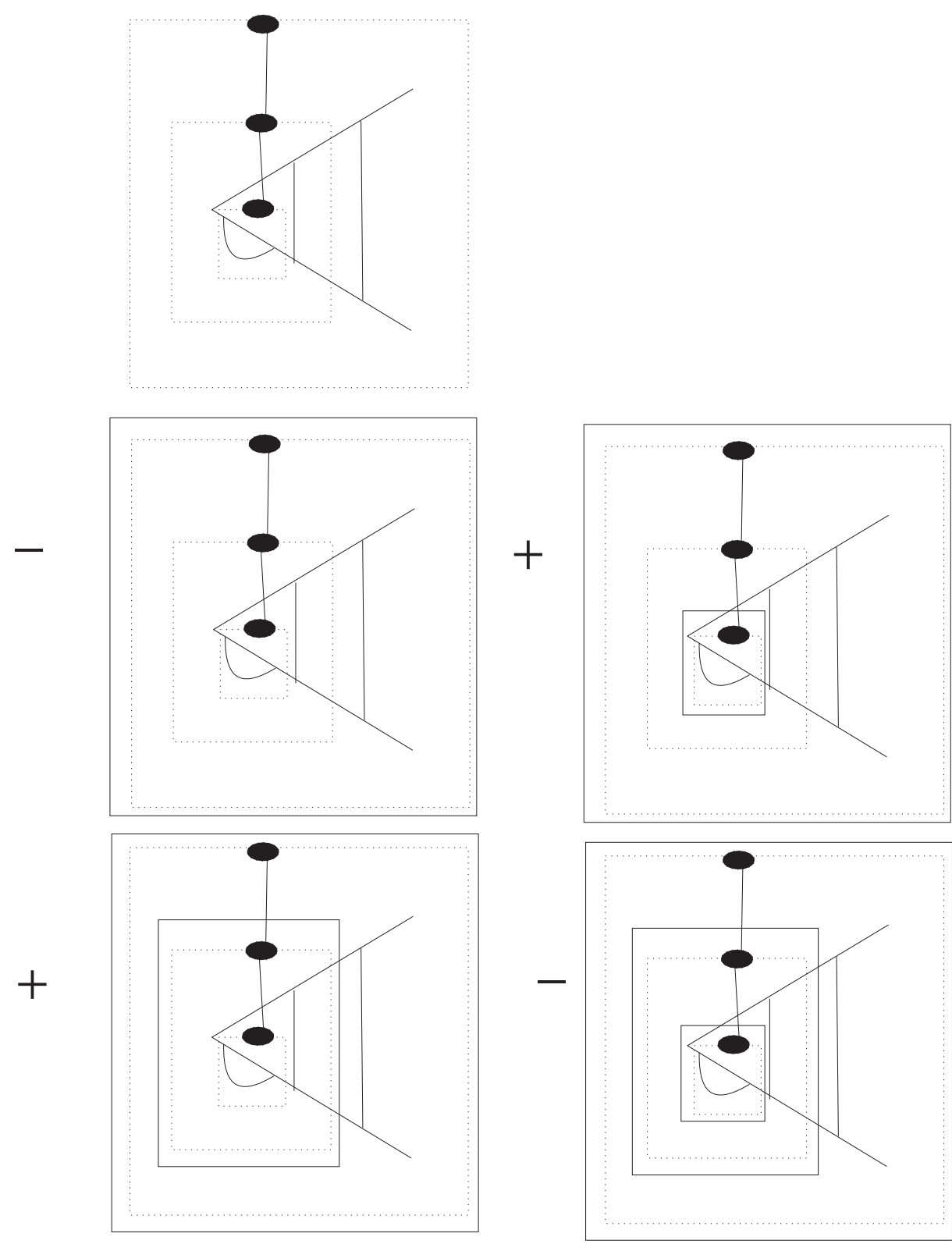

Figure 2: The Hopf algebra of renormalization. We indicate how to assign a decorated tree to a diagram. On such trees we establish the above Hopf algebra structure. Each black box corresponds to a cut on the tree, and these cuts are in one to one correspondence with the forest structure. We calculate the antipode on the tree, and represent the results on Feynman diagrams, to find that the antipode corresponds to the local counterterm. 
and this map is induced by the antipode

$$
S\left[T_{\gamma}\right]=-T_{\gamma}-\sum_{\gamma^{\prime} \subset \gamma} S\left[\gamma^{\prime}\right] \gamma / \gamma^{\prime} .
$$

Hence, in accordance with [3] we find the $Z$-factor of a graph $\gamma$ as derived from the antipode in the Hopf algebra of rooted trees.

\section{Conclusions}

What is now the message which we can learn from this structure of perturbative QFT? It seems to me that the only message is that we shall be not so certain in which function space to look for classical solutions. The above described mathematics relate local QFT to functional analysis and noncommutative geometry. The question to which extent undetected high frequency components are allowed to contribute is of direct relevance for the space of solutions considered, and a mathematical rigorous treatment will have to explore the mathematics of functional analysis and operator algebras, the very disciplines in which a local QFT has its basis anyhow. The correct asymptotic behaviour of a local QFT, characterized by the unimportance of high frequency components, will have meaning for the limit to classical field theory. A quantification of this statement and hence a derivation of the Nimtz anomaly will have to wait until our understanding of QFT has reached maturity.

\section{Acknowledgements}

It is a pleasure to thank the organizers of the $v>? c$ workshop for support and hospitality.

\section{References}

[1] A. Enders, G. Nimtz, J.Phys.I (France), 2 (1992); G. Nimtz, W. Heitmann, Prog.Quant.Elec.21, 81 (1997).

[2] F.E. Low, talk given at this workshop; F.E. Low (MIT, LNS), P.F. Mende, Annals Phys.210, 380-387 (1991).

[3] D. Kreimer, Adv.Theor.Math.Phys.2, 303-334 (1998), q-alg/9707029,

A. Connes, D. Kreimer, Hopf Algebras, Renormalization and Noncommutative Geometry, IHES-M-98-60, to appear in Comm.Math.Phys., hepth/9808042;

D. Kreimer, How useful can knot and number theory be for loop calculations, talk given at Rheinsberg 98, to appear in Act.Phys.Pol., hep-th/9807125. 